Stocker, B. A. D. (1956). J. gen. Microbiol. 15, 575-598

\title{
Abortive Transduction of Motility in Salmonella; a Non- replicated Gene Transmitted through Many Generations to a Single Descendant
}

\author{
BY B. A. D. STOCKER \\ The Guinness-Lister Unit, Lister Institute of Preventive Medicine, \\ Chelsea Bridge Road, London, S.W. 1
}

SUMMARY: Cultures of non-motile Salmonella strains treated with phage lysatts of motile strains produce in semi-solid agar: (i) swarms, each consisting of a clone of motile bacteria, attributed to complete transduction of motility; (ii) trails, i.e. unbranched linear groups of microcolonies stretching some millimetres from the site of inoculation, attributed to abortive transduction of motility; the latter was thought to result from the importation by phage of a gene conferring motility, which was not replicated but was transmitted, as a supernumerary gene, down one line of descent (unilinearly) to but one of the descendants of the organism which received it, the trail marking the path of the gene-bearing descendants of successive generations.

The present work has led to a modified hypothesis: that the phage-imported non-replicated gene confers on the organism the ability to synthesize motility conferring (MC) particles, which are distributed amongst its non-gene-bearing progeny, which cannot make new ones; that each particle may be unilinearly transmitted for many generations; and that while one MC particle confers motility in broth, several are needed to enable a bacterium to travel in a semi-solid medium.

The main evidence for this hypothesis is provided by pedigrees of bacteria made motile by abortive transduction, isolated by micromanipulation from treated suspensions. All such bacteria produce mainly non-motile offspring. A minority (exceptional or $E$ bacteria) produce clones which after 10-15 generations include 20 to 100 motile descendants; the rest give clones containing only a few (0-12) motile organisms. $E$ bacteria are identified as ones containing the supernumerary gene, the others as ones motile through possession of a few MC particles only.

In each of six extensive pedigrees a single $E$ bacterium was isolated from amongst the 9th-21st generation progeny of the original $E$ parent. None of a large number of other motile bacteria isolated in collateral sublines (and so ex hypothesi not gene-bearing) produced more than 12 motile descendants. These data show that the $E$ character (ability to produce 15 or more motile descendants) is unilinearly transmitted.

Any motile bacteria found amongst the progeny of a non- $E$ bacterium after six or more generations were inferred to be motile through possession of one MC particle each; of many such which were isolated none produced more than one motile descendant; when this in turn was isolated, a single motile organism was sometimes again detected in the clone produced. This shows that there is unilinear transmission of motility (in broth).

Samples of populations of motile bacteria isolated by micro-manipulation from treated suspensions were transferred to semisolid agar, or to individual droplets; the proportion of bacteria which generated trails was about equal to the proportion found to be $E$. This was to be expected if both characters reflect the presence of the non-replicated gene.

The MC particle is probably a flagellum, or a granule which determines the production of one. The particle presumed to account for the unilinear transmission of the $E$ and trail-forming characters is most economically interpreted as a phage-imported fragment of genetic material which has failed to replace its homologue in the genetic organelle of the recipient organism. 
Many different hereditary properties may be transferred from one Salmonella strain to another by applying phage lysates of one strain to a culture of the other; this transfer of hereditary characters (transduction) is believed to result from the transfer by phage of genes or fragments of genetic material from organisms of the lysed strain to organisms of the recipient strain (Zinder \& Lederberg, 1952; Stocker, Zinder \& Lederberg, 1953; Lederberg \& Edwards, 1953 ; etc.). Transduction of characters is inferred from the detection of a clone showing the transferred character, and organisms of such clones transmit the new character to all their descendants. The inheritance of the new character by all the progeny of a transformed organism and the total disappearance of the alternative character which was present before treatment are striking features, both of the transduction of characters in Salmonella and of the analogous phenomenon of transformation of hereditary characters in pneumococcus, etc., mediated by the application of deoxyribonucleic acid (DNA) extracted from an appropriate strain. In both systems a proposed explanation is that extraneous genetic material regulating the new character has entered the recipient organism and replaced the homologous genetic material which determined the old character, the latter material being lost. There is evidence for linkage of some of the transferable genetic factors, which suggests that these factors normally form part of a differentiated genetic organelle, perhaps analogous to a chromosome; if extraneous genetic material became incorporated into such a structure it would presumably be thereafter replicated and transmitted to the progeny by whatever mechanisms effect the replication and transmission of the organism's intrinsic genetic material.

In the course of experiments on the transduction of motility from motile (wild-type) to non-motile Salmonella strains (Stocker et al. 1953) a phenomenon was encountered which suggested that some bacteria which became motile by transduction produced non-motile progeny. Such unstably transformed bacteria were ascribed to 'abortive transduction', in contrast to complete transduction which produces stably transformed ones. Its occurrence was inferred from the behaviour of certain non-motile strains when incubated on a semi-solid medium. Untreated cultures produced surface growth, strictly confined to the site of inoculation. Cultures treated with a suitable lysate produced: (i) spreading swarms, each swarm being the motile progeny of a completely transformed organism; (ii) groups of micro-colonies in the depths of the medium, the colonies of each group being arranged along, or close to, an unbranched line extending several millimetres from the site of inoculation. These groups were called 'trails'. The microcolonies comprising a trail yielded on subculture only non-motile growth, similar to that of the parent strain; yet their situation showed that each must have grown from a parent which had moved through the semi-solid medium. It was suggested that each trail arose from a bacterium made motile by abortive transduction; and that when this divided it produced one non-motile daughter, which formed a microcolony in situ, and one motile daughter which travelled on through the semisolid medium, and itself repeated the process when it divided, and so on at each successive division. 
Such abortive transduction of motility was tentatively ascribed to introduction by phage into a non-motile recipient of a normal or wild-type gene which failed to displace from the genetic organelle its homologue determining non-motility, but which caused the recipient organism to become motile despite the simultaneous presence of this homologue; and it was suggested that such a supernumerary gene would not be replicated, so that at division it would pass to only one of the daughters. In this model an organism having a certain character transmits this character (and the factor or particle determining it) down a single line of descent to but one of its progeny in any generation; this will be called 'unilinear transmission'. Fig. 1 illustrates the original hypothesis that trail formation results from the unilinear transmission of motility amongst the progeny of a bacterium abortively transformed to motility. This paper describes experiments designed to test this hypothesis, by direct observation of the motility of the descendants of motile bacteria isolated by micromanipulation from lysate-treated suspensions. Professor $\mathbf{J}$. Lederberg (University of Wisconsin) has also investigated this problem by micromanipulation techniques, and I have had the benefit of frequent discussion and reports of the progress of his work. However, our materials, experimental results and conclusions differ to some extent and we are therefore reporting them separately (see Lederberg, 1956).

The nature, and indeed the existence, of the non-replicated determinant postulated to account for abortive transduction is uncertain, but for brevity it will be rèferred to as a 'supernumerary gene' and the organism presumed to contain it will be called the 'gene-bearing' organism; the justification for this description will be discussed later.

It soon became evident that the hypothesis in its simple form could not account for the results obtained. In the first place, counts of colonies in trails indicated that during the formation of a trail several micro-colonies were initiated per generation time, instead of the one predicted by the original theory. Secondly, single motile rods isolated by micro-manipulation from phage-treated cultures were found to produce, after many generations, progeny comprising a large number of non-motile together with several motile bacteria, instead of the single motile descendant predicted by the original theory. These discrepancies might be explained by 'phenotypic lag'. Normal (wild-type) strains of Salmonella are motile by means of flagella, of which each bacterium has several. The non-motile strains used in the present experiments lack flagella, which is presumably why they are non-motile. A bacterium which becomes motile by abortive transduction must owe its motility to an acquired ability to produce one or more flagella. When it divides, the daughter which does not receive the supernumerary gene perhaps receives some part of the locomotor apparatus of the parent, e.g. some of its flagella or some of the enzymes concerned in their synthesis, so that this daughter is motile, at least for a time. From what is known of phenotypic lag in other situations it might be supposed that an organism which was thus motile only because it contained 'products of gene action' would produce descendants whose motility would decrease at each successive generation, until finally all were non-motile. As will be shown, 
micromanipulation experiments gave results incompatible with this supposition, but explicable by the following hypothesis.

Hypothesis of motility conferring particles, transmitted unilinearly amongst progeny. This hypothesis supposes that the presence of the supernumerary gene in an organism causes it to produce stable particles, which are distributed amongst the daughters at division, and that the presence of even a single

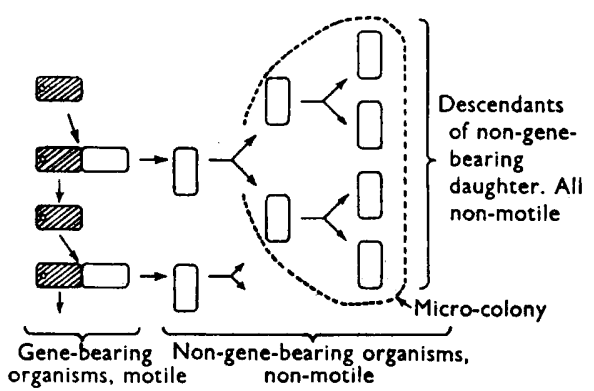

Fig. 1

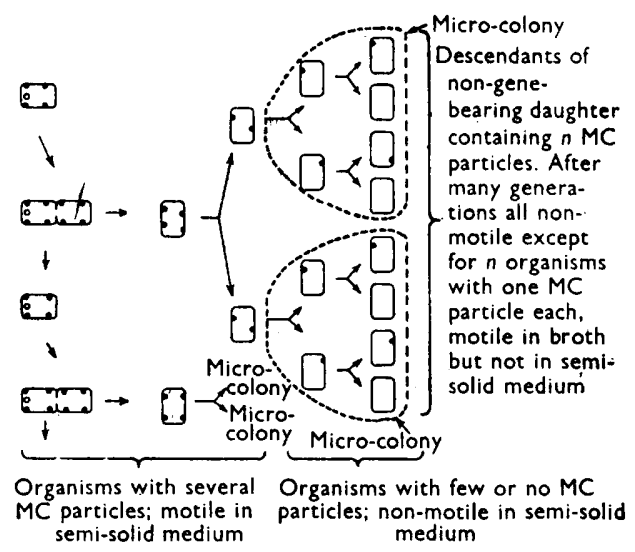

Fig. 2

Fig. 1. Original hypothesis of trail formation; unilinear transmission of supernumerary gene which confers motility. Each division of gene-bearing organism produces (i) a genebearing daughter which moves on, (ii) a non-gene-bearing daughter which is nonmotile and produces a micro-colony of the trail. One micro-colony initiated at each generation. Only one motile descendant of gene-bearing organism. $O$, supernumerary gene; a motile organism; $\square$, a non-motile organism.

Fig. 2. Final hypothesis of trail formation; unilinear transmission of supernumerary gene which confers ability to generate motility-conferring (MC) particles, which are themselves unilinearly transmitted. Each division of gene-bearing organism produces (i) a gene-bearing daughter (with several MC particles) which moves on, (ii) a non-genebearing daughter which contains several MC particles (4, in this diagram) and in consequence moves through the semi-solid medium; after some generations the number of MC particles/organism amongst its descendants falls below some critical level $(2$, in this diagram) so that each ceases to move, and initiates a micro-colony of the trail. Several micro-colonies (2, in this diagram) initiated at each generation. Unilinear transmission of each MC particle, and so of 'motility in broth'; gene-bearing organism produces many descendants motile in liquid (though not in semi-solid) medium. $\bigcirc$, supernumerary gene; $\bullet$, motility-conferring particle.

particle in a bacterium confers on it some degree of motility. The progeny of a motile but non-gene-bearing organism will then include after a time several descendants, each containing a single motility-conferring particle; each such organism will transmit its particle, and hence a degree of motility, to one of its descendants. The hypothesis thus postulates two orders of unilinear transmission, the primary line of transmission being that of the supernumerary gene, whose presence in an organism results in the production of motility conferring particles, each of which is transmitted down a secondary line. To account for the absence of branching in trails it is necessary to postulate that 
only bacteria which contain several particles can travel through semi-solid agar, though a bacterium with one particle is motile in broth. This hypothesis, and the way it accounts for trail formation, is illustrated in Fig. 2. The motility conferring particles are probably flagella, or granules each of which determines the production of a flagellum, but as this is unproven, they will be referred to only as motility conferring (MC) particles.

\section{METHODS}

Organisms. The phage used and most of the bacterial strains were those of Stocker et al. (1953). SW 543, an O strain of Salmonella paratyphi-B, was the parent strain of sublines: SL 160, used in a few of my experiments; and SW 666, used by Lederberg (1956). For the present purpose both sublines may be regarded as identical with strain SW 543. SW 966 is a Salmonella 0 strain of group B (strain 3010-49 of Dr P. R. Edwards), identified as $S$. paratyphi-B by Lederberg (personal communication). Most of the micro-manipulation experiments were made on strain SW 541, a S.typhimurium 0 strain which has never been observed to mutate spontaneously to motility, and which yields large numbers of trails (and swarms) when treated with a suitable lysate. Strain TM2, a motile $S$. typhimurium, was generally used as donor strain. In experiments on $S$. paratyphi-B strain SL 160 lysates of a motile derivative of this strain were used; such lysates are more effective than $S$. typhimurium lysates (Lederberg, personal communication). In all cases the lysates were made with phage PLT 22.

Treatment of non-motile cultures with phage lysates. One volume of a broth culture of a non-motile strain was mixed with an equal volume of a bacteriologically sterile lysate with a phage titre of at least $3 \times 10^{\circ}$ particles $/ \mathrm{ml}$., and the mixture was incubated at $37^{\circ}$. For observations on trails, samples of this mixture were plated after $30 \mathrm{~min}$. on to a semi-solid gelatin agar medium (Stocker et al. 1953), and the plates were incubated for 12-18 hr. at 37 ${ }^{\circ}$. To avoid the trails being obscured the mixture was so diluted that most plates did not develop any swarms. For determining the number of micro-colonies arising in a trail per hr. of incubation at $37^{\circ}$, the plates were incubated for about $12 \mathrm{hr}$. at $37^{\circ}$, and then held at room temperature overnight. This second period allowed the minute micro-colonies at the distal end of the trail to grow to a visible size; the solidification of the gelatin-containing medium prevented the initiation of any new colonies by motile bacteria.

For the isolation of motile bacteria by micromanipulation, the mixture of bacteria and phage was incubated for 90-120 min. at $37^{\circ}$ before transfer to the micromanipulation chamber. In some experiments many of the bacteria isolated failed to grow; this mortality was largely avoided by using broth cultures near the end of the log phase in place of fully grown cultures, and by diluting the phage-treated culture with broth at intervals during the period of incubation. The results of micromanipulation experiments were generally similar, whether log-phase or overnight cultures were used.

Micro-manipulation. A Singer micromanipulator (Barer \& Saunders-Singer, 
1948) was used. Angled micropipettes were made by the dropping-weight method (de Fonbrune, 1949). Micromanipulation methods generally were based on those of de Fonbrune (1949). Drops of broth were deposited on the lower surface of a coverslip which formed the roof of a chamber filled with liquid paraffin. It was not convenient to use a fresh micropipette for each operation; it was found that when only one rod was taken up at a time, the sterility of the pipette could be assumed when a single rod (of about the same size) had been expelled. Observations were made by low-power darkground microscopy, with an $8 \mathrm{~mm}$. objective of N.A. 0.5 and a long-working-distance Cooke, Troughton and Simms phase-contrast condenser unit, with the annulus intended for the $2 \mathrm{~mm}$. objective.

For isolation of motile bacteria, a drop of phage-treated culture was placed at one point of the coverslip, and droplets of sterile broth at others. Motile rods frequently travelled round the periphery of the drop, whereas the non-motile ones tended to sediment into the concavity of the drop, leaving the border free, so that it was generally easy to pick up single motile rods from the edge of the drop, even when they were present in a proportion of $1: 10^{4}$ or less. This operation was facilitated by the use of a 'trapping drop', that is a drop of sterile broth placed so that it just came into contact with the drop containing the phage-treated culture. Motile bacteria swam out into this sterile broth, and could be isolated very easily.

Motile rods thus isolated were transferred to the previously prepared broth droplets. The oil-chamber was kept at room temperature, and re-examined at intervals; and the number of motile and non-motile rods in each droplet was estimated and recorded. Rods which were spinning or twitching, movements which were obviously not Brownian, were recorded as motile. Even when a droplet contained very large numbers of non-motile bacteria ones in translational movement were detected with surprising ease, partly because of their tendency to travel round the periphery of the drop (Pl. 1, figs. 3, 4). When more than about eight motile rods were present in a drop it was difficult to count them exactly, except by removing them one by one; counts recorded as greater than eight are therefore for the most part estimates. In the pedigree experiments motile bacteria amongst the progeny were re-isolated from the droplets to fresh droplets, the large numbers needed being set out in grid formation by the use of the mechanical stage; it was found necessary to interrupt periods of observations at room temperature by refrigerating the oil chamber at $c .4^{\circ}$ for up to $24 \mathrm{hr}$., but this did not seem to affect the subsequent behaviour of the organisms.

\section{OBSERVATIONS ON TRAILS}

The majority of about 20 non-motile Salmonella strains tested produced trails as well as swarms when treated with lysates; the ratio of trails to swarms varied from about $1: 1$ to $100: 1$ or more. Stocker et al. (1953) found that a non-motile strain treated with a lysate of some other non-motile strain commonly produced swarms, which was taken to indicate non-homology of the genetic factors causing non-motility in the two strains; many such combina- 
tions yielded trails also. In contrast, treatment of a culture of a non-motile strain with a lysate of that same strain never evoked trails (or swarms). Some non-motile strains when grown on a semi-solid medium spontaneously produce microcolonies just below the inoculated surface of the medium (Stocker et al. 1953); but these spontaneous satellite microcolonies were never arranged linearly, like the microcolonies of a trail.

In most strains the trails were short, and did not increase in length when incubation was continued for longer than $18 \mathrm{hr}$. The spontaneous termination of a trail presumably indicates that after a certain number of generations there is no longer present, amongst the descendants, a viable organism containing the supernumerary gene. However, a non-flagellated strain of Salmonella paratyphi-B, SW 966, produced trails which after $42 \mathrm{hr}$. of incubation at $37^{\circ}$ were about $20 \mathrm{~mm}$. long (Pl. 1, Figs. 1, 2). No branching was seen, even in these long trails. They seemed always to extend laterally, more or less directly away from the site of inoculation, which suggests a negative chemotactic response to some product of bacterial metabolism.

Counts of the number of colonies initiated per hour of incubation were made by the method described above. Lysate-treated cultures of Salmonella typhimurium strain SW 541 incubated at $37^{\circ}$ for $15 \mathrm{hr}$. produced trails made up of 80-90 colonies. Its generation time at this temperature is $20-30 \mathrm{~min}$., so at most 45 generations could have elapsed during this time. Thus an average of at least two colonies were initiated at each division of the gene-bearing organism; this indicates that phenotypic delay in loss of ability to move through gelatinagar lasts between one and two generations at least. The colonies forming a trail were arranged in pairs and small groups, which became confluent as they enlarged; the calculated minimum of two colonies per generation is therefore probably an underestimate.

On two occasions a swarm appeared to have arisen at the distal end of a trail several mm. long; but the strains concerned were known to undergo spontaneous mutation to motility at fairly high rates. From the rarity of such appearances it is inferred that a transformation which is not completed within the first few generations is seldom or never completed thereafter.

\section{ISOLATION OF MOTILE ORGANISMS FROM PHAGE-TREATED SUSPENSIONS}

Motile bacteria were isolated by micromanıpulation, by the method described above, from lysate-treated suspensions of Salmonella typhimurium strain SW 541. Most of them became immobilized either at the glass or at the oil interface soon after they had been transferred to individual droplets. This accidental immobilization generally made it impossible to determine motility in the organisms resulting from the first divisions of the originally isolated bacterium. Because of it, counts of actually moving bacteria give only minimum estimates of the number of potentially motile ones present. Droplets each inoculated with a single motile rod were examined at intervals, to ascertain the number of motile and non-motile descendants. 
The most informative results were obtained after overnight incubation, by which time each droplet contained some hundreds or thousands of bacteria. At this time the results predicted by the hypothesis of MC particles are as follows (a motile bacterium isolated from a lysate-treated culture will be called an 'initial'). There are three kinds of 'initial':

(i) Immediate descendants of gene-bearing organisms, themselves lacking the supernumerary gene but motile because of the presence of a few (say $n$ or less) MC particles, obtained from the parent. These particles will be distributed amongst the offspring; after 10 generations the descendants will number c. 1000, of which only $n$ or less will be motile, through the presence in each of one MC particle.

(ii) Gene-bearing organisms. An organism of this sort divides to give a gene-bearing daughter, and a non-gene-bearing daughter which contains a few (say an average of $n$ ) MC particles; the gene-bearing daughter in turn divides in the same way; and the process is repeated in each generation. In consequence after 10 generations there will have been produced in all about $10 n$ particles. As those MC particles produced in the earlier generations will have been distributed, there will be present, in addition to a majority of non-motile descendants, one gene-bearing motile descendant and nearly $10 n$ descendants motile by possession of MC particles.

(iii) Completely transformed organisms, and their descendants. These will produce clones of motile bacteria.

Thus the hypothesis predicts that the populations resulting from $c .10$ generations of growth of 'initials' will be of three kinds: (i) mainly non-motile, with a few motile; (ii) mainly non-motile with many motile; (iii) all motile.

In a series of experiments on Salmonella typhimurium strain SW 541 (Table 1, Expts. i-vi), growth was obtained from 152 out of 164 'initials' isolated. Five populations of motile rods were inferred to have grown from completely transformed 'initials'. The remaining 147 populations, made up predominantly of non-motile rods, could be divided into two classes with, respectively, few and many motiles, namely: 116 populations with 0-12 motile rods; and 31 populations with 19-80 motile rods. The former class includes 16 populations in which no motile organisms were seen; these are interpreted as populations in which the MC particles responsible for the motility of the 'initial' have escaped detection.

It was at first supposed that populations containing many (e.g. 50) motile rods resulted from the delayed completion of an abortive transformation, resulting in the production of a small clone of fully transformed bacteria. This was not the case, since numerous motile rods isolated from such populations always produced progeny which were nearly all non-motile.

'Initials' producing populations containing many motile rods were always a minority, generally about a fifth of the total. These 'initials' are interpreted as gene-bearing; but as a non-committal label any organism giving rise to more than 15 motile descendants amongst a predominance of non-motile ones will hereafter be termed $E$ (exceptional).

In a further series of experiments the immediate progeny of the 'initials', 


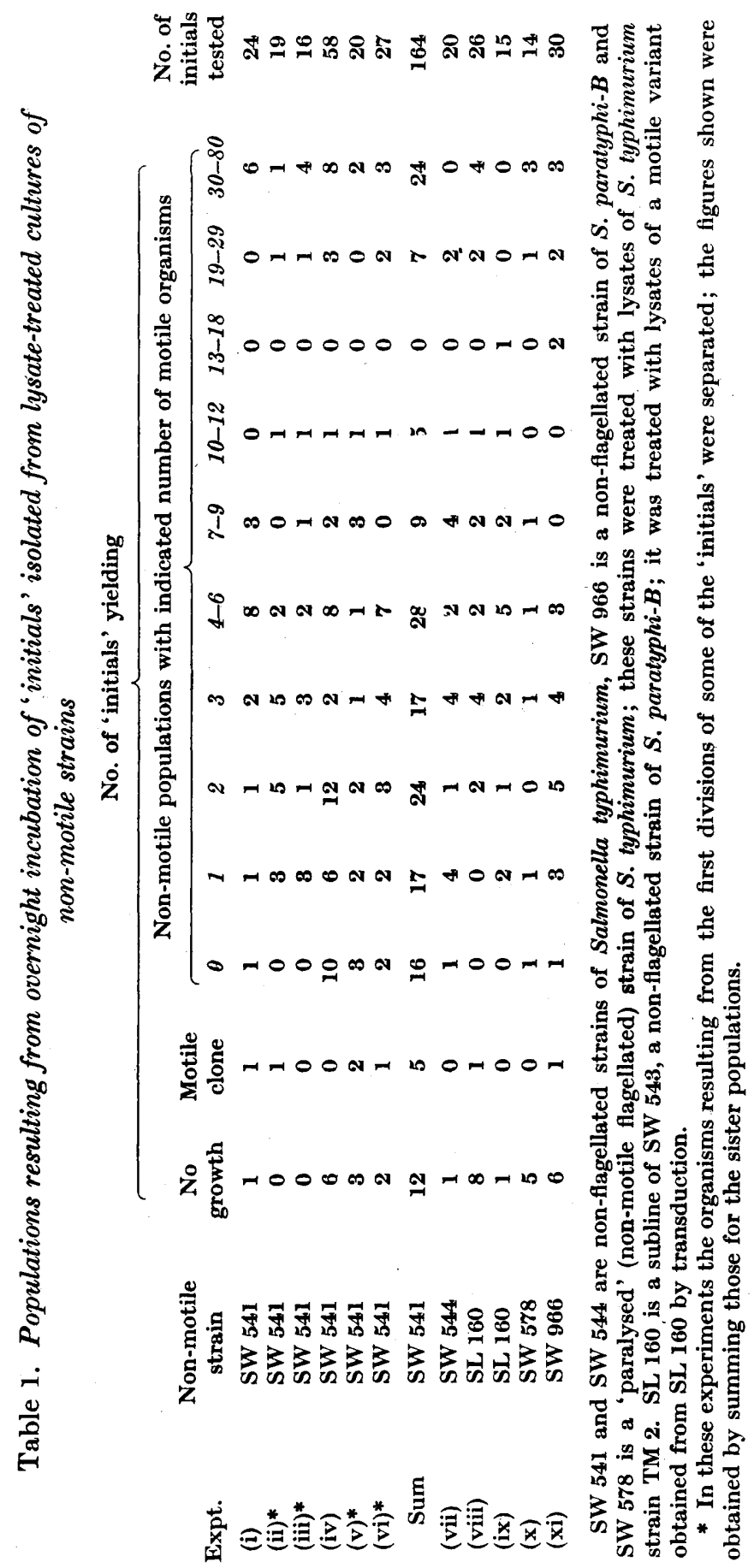

G. Microb. xv 
when they numbered one to six, were transferred to separate droplets; after overnight incubation the resulting sets of sister populations were examined, and the numbers of motile descendants detected were recorded (Table 2). It will be seen that when the total number of motile descendants of an 'initial' numbered less than 15 they were distributed more or less equally amongst the sister populations of a set; as these 'initials' are interpreted as organisms

Table 2. Numbers of motile descendants detected in progenies of sister organisms produced by division of 'initials'

'Initials' isolated from lysate-treated cultures of Salmonella typhimurium strain SW 541 were allowed to divide. The resulting organisms, when they numbered 2 to 6 , were isolated in separate droplets. After overnight incubation the resulting sister populations were examined; all contained hundreds or thousands of non-motile organisms, as well as the motile ones recorded.

\begin{tabular}{|c|c|c|c|c|c|c|c|c|}
\hline \multirow{2}{*}{$\begin{array}{c}\text { 'Initial' } \\
\text { no. } \\
1\end{array}$} & \multirow{2}{*}{$\begin{array}{c}\text { Sisters } \\
\text { separated } \\
\text { when they } \\
\text { numbered } \\
\text { Two }\end{array}$} & \multicolumn{6}{|c|}{$\begin{array}{l}\text { No. of motile progeny seen in the } \\
\text { resulting sister populations }\end{array}$} & \multirow{2}{*}{$\begin{array}{c}\text { Total } \\
\text { no. of } \\
\text { motile } \\
\text { progeny } \\
\text { seen } \\
0\end{array}$} \\
\hline & & 0 & 0 & . & . & . & . & \\
\hline 2 & Two & 0 & 1 & . & . & . & . & 1 \\
\hline 3 & Two & 0 & 2 & . & . & . & . & 2 \\
\hline 4 & Two & 1 & 2 & . & . & . & . & 3 \\
\hline 5 & Two & 1 & $\mathbf{2}$ & . & . & . & . & 3 \\
\hline 6 & Two & 1 & $\overline{2}$ & . & . & . & . & $\mathbf{3}$ \\
\hline 7 & Two & 3 & 6 & . & . & . & . & $\theta$ \\
\hline 8 & Two & 5 & 6 & . & . & . & . & 11 \\
\hline 9 & Two & $\mathbf{3}$ & c. $\mathbf{3 0}$ &. & . & $\dot{0}$ & $\dot{0}$ & c. $\mathbf{3 3}$ \\
\hline 10 & Two & $\mathbf{3}$ & c. 40 & . & . & . & . & c. 43 \\
\hline 11 & Three & $*$ & 0 & 2 & . & . & . & 2 \\
\hline 12 & Three & 0 & 1 & 8 & . & . & . & $\boldsymbol{\theta}$ \\
\hline 13 & Three & $\mathbf{2}$ & 3 & 29 & . & . & . & 34 \\
\hline 14 & Four & $*$ & o & o & 2 & . & . & 2 \\
\hline 15 & Four & $\mathbf{0}$ & 0 & 1 & 1 & . & . & 2 \\
\hline 16 & Four & 0 & 1 & 2 & 2 & . & . & 5 \\
\hline 17 & Five & 2 & 2 & $\mathbf{3}$ & 5 & 17 & . & 29 \\
\hline 18 & Six & * & * & $\mathbf{0}$ & 0 & 1 & 2 & $\mathbf{3}$ \\
\hline 19 & Six & $\mathbf{0}$ & o & 0 & 1 & 1 & 3 & 5 \\
\hline 20 & Six & o & 0 & 0 & $\mathbf{3}$ & 4 & c. 50 & c. 57 \\
\hline 21 & Six & o & 0 & 1 & 2 & 5 & c. 65 & c. 73 \\
\hline
\end{tabular}

motile only through the presence of $\mathrm{MC}$ particles it is inferred that $\mathrm{MC}$ particles are distributed about equally amongst the daughters at division. In the case of $E$ 'initials', producing in all more than 15 motile descendants, these were distributed very unevenly, nearly all being found in only one of a set of sister populations. For instance, all but 8 of the $c .73$ motile progeny of ' initial' no. 21 were descendants of one of the six organisms resulting from the first divisions of the 'initial'. On the hypothesis adopted 'initial' no. 21 was a gene-bearing organism, and the sister population containing many motile descendants was derived from the single gene-bearing organism of the six into which it divided. 


\section{Abortive transduction of motility}

If all the descendants of an organism motile only through presence of products of gene action were non-motile after a period of phenotypic lag of less than 10 generations, then in experiments of this sort motile descendants would be found in no more than one of a set of sister populations, viz. that derived from the gene-bearing sister.

\section{PEDIGREE EXPERIMENTS}

The best evidence obtained for the postulated two different orders of unilinear transmission comes from experiments in which the transmission of the 'supernumerary gene' was followed through many generations. To obtain such pedigrees, a number of motile 'initials' were isolated as before, and the organisms resulting from their first few divisions were separated. Some generations later an attempt was made to select families which had grown from

Table 3. Summary of pedigrees of six 'initials' isolated from lysate-treated cultures of Salmonella typhimurium strain SW 541

\begin{tabular}{|c|c|c|c|c|c|}
\hline \multirow[b]{2}{*}{$\begin{array}{c}\text { Pedigree } \\
\text { no. }\end{array}$} & \multirow[b]{2}{*}{$\begin{array}{c}\text { Intermediate } \\
\text { isolations of } \\
E \text { descendant } \\
\text { at generation } \\
\text { no.* }\end{array}$} & \multicolumn{2}{|c|}{ No. of motile descendants of } & \multirow{2}{*}{$\begin{array}{l}\text { Generations } \\
\text { between 1st } \\
\text { and last } \\
\text { isolation of } \\
E \text { organism } \\
\text { (g) }\end{array}$} & \multirow[b]{2}{*}{$\begin{array}{c}\text { Motile } \\
\text { descendants } \\
\text { per } \\
\text { generation } \\
(a / g)\end{array}$} \\
\hline & & $\begin{array}{c}\text { last- } \\
\text { isolated } \\
E \text { descendant }\end{array}$ & $\begin{array}{l}\text { all its } \\
\text { collateral } \\
\text { relatives } \\
(a)\end{array}$ & & \\
\hline $1 a$ & $4,10,13$ & c. 100 & 85 & 20 & $4 \cdot 2$ \\
\hline $\mathbf{3 a}$ & $2, \mathbf{3}, \mathbf{9}$ & c. 88 & $\mathbf{2 7}$ & $\mathbf{9}$ & $\mathbf{3 \cdot 0}$ \\
\hline $10 a$ & $2,3,4,13$ & c. 30 & $40 \dagger$ & $11 \dagger$ & $\mathbf{3 . 5}$ \\
\hline $21 y$ & $2,4,6$ & c. 35 & 28 & 8 & $3 \cdot 5$ \\
\hline $25 a$ & $3,8,12,13$ & c. 20 & $\mathbf{5 5}$ & 13 & $4 \cdot 2$ \\
\hline \multirow[t]{2}{*}{$27 a$} & $2,6,8, y$ & c. 40 & 29 & $\mathbf{9}$ & $3 \cdot 2$ \\
\hline & & & 264 & 70 & $3 \cdot 8$ \\
\hline
\end{tabular}

* Generations calculated from estimated population sizes, the 'initial' being taken as generation no. 1 .

$\dagger$ The first two generations in pedigree $10 a$ have been omitted in this calculation, because of death of several organisms.

$E$ 'initials'; such families were recognized by the presence of a relatively large number (8-20) of motile organisms in the family as a whole; their unequal distribution within a set of sister populations sometimes permitted recognition of one of the set as that likely to contain the gene-bearing descendant. All detectable motile rods were isolated from such droplets, in order, if possible, to re-isolate the gene-bearing organism; this process was repeated at intervals. In some pedigrees motile cells which were inferred to be non-genebearing were also isolated. Some experiments of this kind failed, none of the motile descendants isolated after some generations of growth proving to be $E$ (i.e. producing more than 15 motile progeny). However, in six pedigrees the transmission of the $E$ character, and so by inference of the gene, was followed for 8-20 generations (Table 3).

Three of these pedigrees are set out in Fig. 3. For clarity, only some of the transfers and observations made have been included. The estimated numbers 

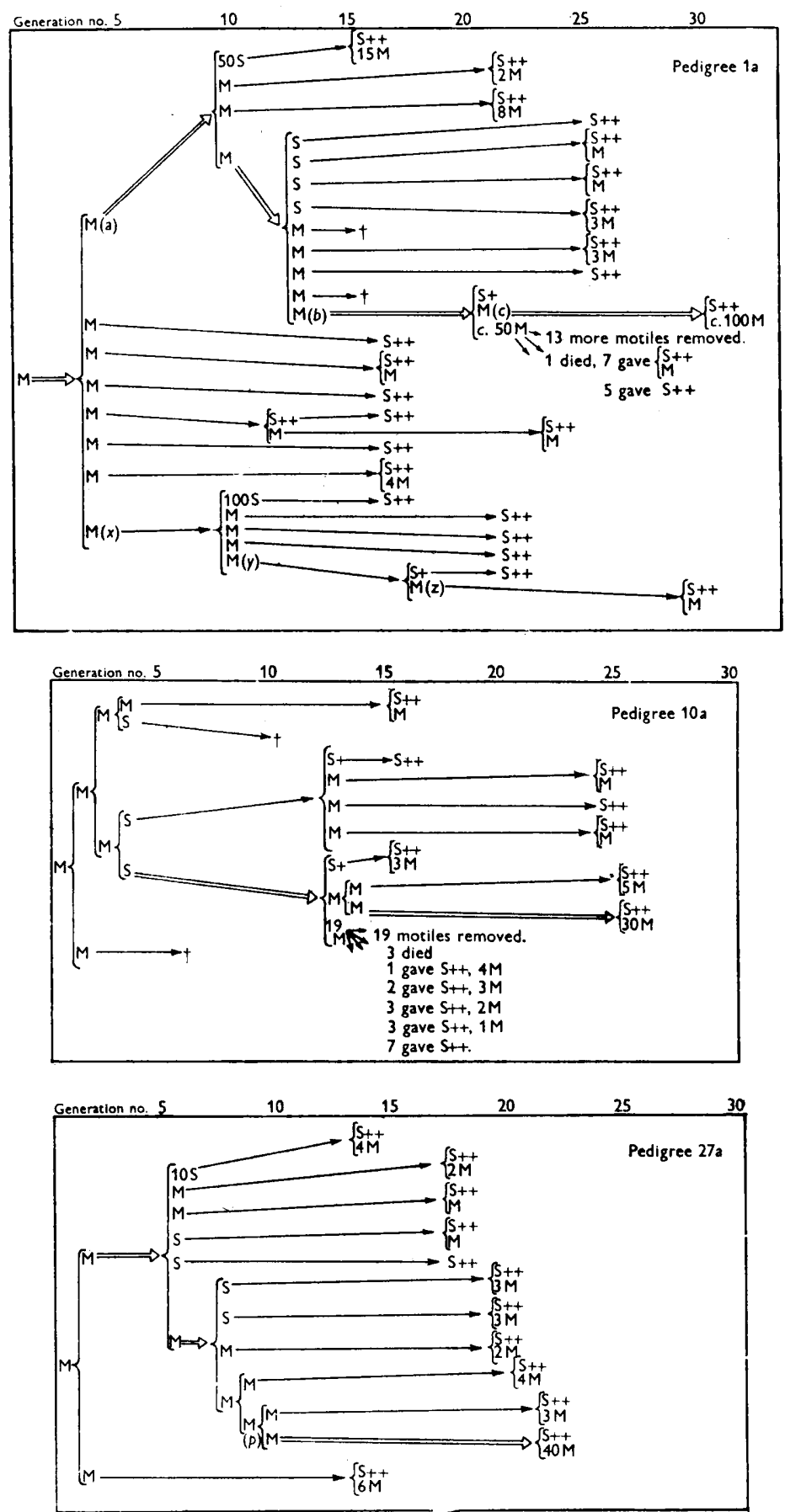

Fig. 3. Pedigrees of three $E$ 'initials' isolated from lysate-treated cultures of strain SW 541. In each pedigree the organism on the left of the diagram is the 'initial'. The progeny of an organism are shown in the bracket, or at the head of the arrow, to its right. Number of generations estimated from population size, the 'initial' being taken as generation 1. M, a motile organism; $2 \mathrm{M}$, two motile organisms, etc.; $\mathrm{S}$, a non-motile organism; $\mathbf{S}+$ hundreds of non-motile organisms; $\mathbf{S}++$, thousands of non-motile organisms; $\uparrow$, organism did not grow; $(a),(b)$, etc., organisms referred to in text. Double arrow indicates inferred line of transmission of supernumerary gene. 
of motile and non-motile bacteria in a droplet are recorded at each time that bacteria were removed from it. The number of generations elapsing since the isolation of the 'initial' from the treated population has been calculated from the estimated population sizes. For simplicity the final composition for the population of a droplet records the largest number of motile rods known to have been present at any period later than the 7 th generation of growth of the organism inoculated into the drop (exclusive of motile organisms removed to other droplets, which are separately indicated). For instance, when a droplet inoculated with one motile rod was later found to contain $c .400$ non-motile and two motile rods, the result is recorded as ' $\mathrm{S}++, 2 \mathrm{M}$ ', that is 'thousands of non-motiles, two motiles', even if, as sometimes happened, only one, or no, motile rods were detected by the time the population numbered thousands.

\section{Evidence for unilinear transmission of the $\mathrm{E}$ character}

If the $\boldsymbol{E}$ character results from the presence of a non-replicated 'gene', then after many generations of multiplication of an $E$ 'initial' one $E$ descendant would be expected amongst its progeny. In the six pedigree experiments $E$ descendants were isolated from amongst the progeny of the (estimated) 9th, 10th, 10th, 14th, 14th, and 21st generations, respectively. All the ancestors of the last-isolated $\boldsymbol{E}$ bacterium back to the 'initial' are of necessity $\boldsymbol{E}$ also, since their progeny includes the progeny of the final $E$ bacterium. If the $E$ character is transmitted unilinearly, then in any one pedigree no organism other than the last-isolated $E$ bacterium, and its ancestors, can be $E$. In pedigrees $1 a$ and $25 a$ there were isolated, respectively, more than 50 and more than 30 viable motile bacteria which, by this argument, could not have been $E$. None of the resulting 80 populations contained more than nine motile rods. Similar, though less extensive, results were obtained in the other four pedigrees and in other experiments.

All the ancestors of an $E$ organism back to the 'initial' are by definition also $E$; but in the pedigrees some of these ancestors are recognizable as $E$, even without taking into account the motile progeny of the $E$ organisms isolated from amongst their offspring. Consider, for instance, organism $b$ of generation 13 in pedigree $1 a$ (Fig. 3); its progeny included organism $c$, which produced c. 100 motile descendants. But even if organism $c$ had died its ancestor, $b$, would still have been recognized as $E$, for it produced $c$. 50 other motile descendants. In the same pedigree organism $a$ of generation 4 may likewise be recognized as $E$ without taking into account its $E$ descendant of generation 13 (organism $b$ ).

On the hypothesis stated, an $\boldsymbol{E}$ bacterium divides to produce one $\boldsymbol{E}$ daughter, and one non- $E$ daughter which will produce a few $(<15)$ motile progeny. In the six pedigree experiments pairs of sisters of which one proved to be $E$ were separated on twelve occasions. All the twelve non- $E$ daughters produced some (one to nine) motile progeny.

In pedigree $27 a$ (Fig. 3) organism $p$, of the estimated 9th generation, divided to produce two daughters, which had, respectively, 3 and $c .40$ motile descendants; thus the unique $E$ descendant of the 9 th generation 
behaved jusı like an $E$ 'initial' (Table 2), i.e. divided into dissimilar daughters which produced, respectively, few and many motile descendants amongst a predominantly non-motile progeny.

\section{Evidence for unilinear transmission of motility-conferring particles}

The bimodal distribution of numbers of motile cells detected in the progeny of 'initials' (Table 1) suggested that the number of MC particles received by the non- $E$ daughter of an $E$ organism was probably not more than fifteen, and it was inferred that after six generations (when the progeny numbered $2^{6}=64$ ) or later, the proportion of descendants of such an organism which contained more than one MC particle would be small. Any motile descendant seen at this stage might therefore be assumed to contain only one MC particle, and would therefore be expected to transmit this particle, and hence motility, to only one of its progeny. In the six pedigree experiments 66 viable, motile descendants of the 6th or later generation of sisters of $E$ organisms were isolated. All these 66 cells produced populations of non-motile bacteria; and in each of 17 of these populations $(26 \%)$ one motile descendant was detected. Similar results were obtained in other experiments, and in no instance has an organism of this kind been observed to produce more than one motile descendant. Accidental immobilization of the bacterium containing the MC particle may account for the non-detection of the expected single motile descendant in the remaining $74 \%$ of the isolations. This high proportion of failures, however, suggested that, even if an organism of the kind under discussion did on occasion produce two motile descendants, this might not be detected. Two further lines of evidence confirm that only one motile descendant is produced.

The more direct evidence is from observations of the actual moment of division of a motile bacterium which was inferred from its pedigree to be motile through possession of a single MC particle. In experiments on Salmonella typhimurium strain SW 541 such observations were impracticable, since the bacteria nearly always became immobilized at an interface shortly after transfer to separate droplets. Motile bacteria obtained by transduction from $S$. paratyphi-B strain SL 160 are less liable to accidental immobilization, and in pedigree experiments with this strain the division of a motile bacterium inferred to contain one MC particle was watched on several occasions; in each case one daughter moved on unchecked, whilc the other was stationary (or in Brownian motion) from the moment of fission.

Further evidence for the unilinear transmission of motility was given by several experiments in which the solitary motile descendant was re-isolated in series. In pedigree $1 a$ (Fig. 8 ) organism $x$ was inferred to be non-gene-bearing, since its 'cousin', organism $a$, was an $E$ organism. The descendants of organism $x$ when they numbered $c .100$ included four motile organisms, each presumably motile through possession of a single MC particle. These four were isolated; one of them, organism $y$, produced progeny numbering some hundreds amongst which was detected a single motile organism, which could now certainly be inferred to be motile through the presence of one MC particle only. This organism, $z$, in turn produced a clone of non-motile rods amongst which, 
when they numbered thousands, a solitary motile rod was again detected. Such a result seems in itself almost sufficient to establish the occurrence of unilinear transmission of motility and by inference the existence of a unilinearly-transmitted motility;conferring particle.

\section{The number of $M C$ particles received by a non-E daughter of an $\mathrm{E}$ organism}

On the hypothesis adopted, when a gene-bearing organism divides one daughter will receive a few (say an average of $n$ ) MC particles, and will produce a clone containing $n$ potentially motile descendants. In the six pedigrees viable rods later recognized as sisters of $\boldsymbol{E}$ organisms were isolated on twelve occasions; the number of motile rods detected in the resulting clones varied from one to nine (average 4.4). Since not all potentially motile descendants were detected this gives only a minimum estimate of $n$. The counts of numbers of motile descendants depended for the most part on observations made after many generations of multiplication; it seems likely that the probability of thus detecting a potentially motile descendant (i.e. one MC particle) after several generations of unilinear transmission would be about the same as the probability of detecting a motile descendant after several generations of growth of an organism which contained a single MC particle. The latter probability was estimated above as $26 \%$. The true value of $n$ is therefore estimated as $4 \cdot 4 \times 100 / 26=17$. In this calculation only observations on organisms known to be sisters of $\boldsymbol{E}$ organisms have been used.

Other observations in the pedigree experiments can also be used. If it be assumed that the gene-bearing organism grows and divides at the same average rate as the rest of the progeny of an $E$ 'initial' it is possible to estimate $g$, the number of generations between the isolation of the 'initial' and the last occasion on which its $E$ descendant was isolated. A daughter containing an average of $n$ particles is produced at each generation, so that after $g$ generations the progeny of the $E$ 'initial' will comprise one $E$ organism and non-genebearing ones containing amongst them about $n \times g$ particles. Thus, the total number of motile descendants detected in all the collateral lines of a pedigree (i.e. the populations of all droplets except that to which the $E$ descendant was finally transferred) divided by $g$ (the estimated number of generations) will give an estimate of $n$. The data for the six pedigrees are given in Table 3 ; the estimates of $n$ do not differ much, and their mean, $\mathbf{3 \cdot 8}$, is close to the value of 4.4 estimated from sisters of $\boldsymbol{E}$ organisms. Adjustment for the estimated proportion of undetected MC particles gives a value of $3 \cdot 8 \times 100 / 26=14$ for $n$.

\section{EVIDENCE THAT ONLY $E$ ORGANISMS PRODUCE TRAILS}

Trails have been explained above as marking the path through the semisolid medium of the successive bearers of a non-replicated 'gene' which confers ability to synthesize MC particles; and $E$ organisms have also been identified as ones containing this supernumerary 'gene'. If this be correct then, of the motile organisms which result from abortive transduction, only the $E$ organisms should produce trails. As $E$ organisms were recognized in retrospect, by counting their 
motile progeny, it was impossible to transfer known $E$ organisms to semisolid medium in order to test their trail-forming ability. However, the proportion of $E$ organisms was compared with the proportion of trail-forming ones in samples from collections of motile $c$ 'initials' evoked by lysates. 'Initials' were picked from a trapping drop and transferred alternately to separate droplets, which were retained in the chamber, or to a larger drop, which, when it had received a number of bacteria, was transferred to a tube of broth. From this tube drops were inoculated on to semisolid medium. Of 57 ' initials' kept in the chamber 51 grew and produced predominantly non-motile progeny, and 11 of these 51 (c. 22\%) were $E$ (20-60 motile descendants each). After overnight incubation at $37^{\circ}, 21$ of 33 plates of gelatin agar, each inoculated with one drop from the tube containing trapped 'initials', gave no growth; it is calculated from the Poisson formula that the 12 drops which did yield growth contained about 15 viable organisms. In the inoculated areas there developed three trails, comprising, respectively, $c .35,60$ and 67 colonies. Thus a proportion of 3 in about $15($ c. $20 \%)$ of the viable 'initials' from the trapped population produced trails, and $22 \%$ of them were $E$. Pl. 1, fig. 5, shows a trail produced by an 'initial' transferred to semisolid medium. Motile descendants of organisms later diagnosed as sisters (or other collateral relatives) of $\boldsymbol{E}$ organisms are by inference not themselves $\boldsymbol{E}$. A number of such motile organisms were transferred from the micromanipulation chamber to gelatin agar medium; none of them produced a trail.

Many 'initials' transferred to semisolid medium produced groups of two, three or four adjacent microcolonies. These 'initials' were presumably nongene-bearing organisms containing several MC particles, and therefore able to move through the semisolid medium; their immediate progeny would retain this ability until they contained an insufficient number of $\mathrm{MC}$ particles. Counts of colonies initiated at each generation during trail formation had similarly indicated that the non-gene-bearing daughter of a gene-bearing organism produced an average of at least two colonies in semisolid medium.

As $E$ organisms and their close relatives which contained several MC particles appeared to differ from organisms which contained only one particle by their ability to travel through semisolid medium, other differences in degree of motility were sought. No consistent difference in speed was found, but organisms which contained several MC particles seemed less liable to get stuck at interfaces, and, if they did get stuck, to have a greater chance of breaking away later.

\section{PEDIGREES OF 'INITIALS' PRODUCING CLONES OF MOTILE ORGANISMS \\ Unilinear transmission of motility amongst progeny of sisters of completely transformed organisms}

About $4 \%$ of 'initials' isolated from treated suspensions gave, after overnight incubation, populations containing thousands of motile bacteria which on subculture produced motile populations. This result is attributable to complete 
transformation of the 'initial'. When the daughters produced by the first division of an 'initial' had been separated, it was sometimes found that one daughter gave a clone of motile organisms, while the other produced a clone of non-motile ones. This may be explained by assuming that the original bacterium contained two or more identical 'nuclei' or gene-sets and that the phage-imported gene was incorporated into one of these. Thus, when the bacterium divided the daughter which received the transformed gene-set would produce a clone of motile organisms, while the other daughter, which received the untransformed set, would produce a clone of non-motile organisms.

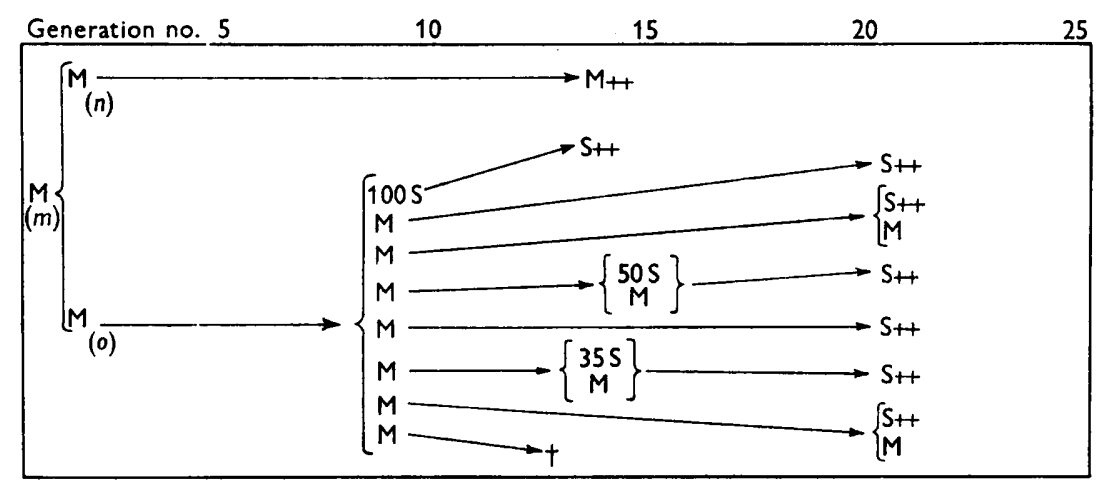

Fig. 4. Pedigree of an 'initial' which produced a clone of motile organisms; the 'initial' (on left of diagram) was isolated from a lysate-treated culture of strain SW 541 . Symbols as in Fig. 3.

Lederberg (1956; personal communication) found that when one of a pair of sister populations consisted of motile bacteria, the other, when it consisted mainly of non-motile ones, yet included a few motile organisms, each of which transmitted motility to only one of its descendants. This was observed in experiments on transduction of motility to Salmonella paratyphi-B strain SW 666. In experiments on $S$. typhimurium strain SW 541 I obtained similar results. For example, in the pedigree shown in Fig. 4 organism $n$ produced a motile clone and its sister, $o$, produced a clone which consisted of $c .100$ nonmotile and 7 motile descendants. The 7 motile organisms were isolated; of the 6 which grew 4 produced clones in which a single motile descendant was seen at some stage between the 6th and 13th generation. It is inferred that organism $o$ contained at least 7 MC particles obtained from its parent, $m$, which was able to form MC particles because a phage-imported gene had been incorporated into one of its originally identical gene-sets. An alternative explanation would be that motility had been abortively transduced to the 'initial', so that it contained a supernumerary gene and as a result formed MC particles, some of which passed to each daughter when it divided; and that the supernumerary gene later became incorporated into the gene-set in the daughter to which it chanced to pass. 


\section{MICROMANIPULATION EXPERIMENTS ON OTHER NON-MOTILE SALMONELLA STRAINS}

Motile bacteria were isolated by micromanipulation from lysate-treated cultures of two non-flagellated Salmonella strains, SW 544 and SW 966, and one non-motile flagellated strain, SW 578; these experiments gave results (Table 1) similar to those described above. Unilinear transmission of ' motility in broth' was observed in pedigree experiments on all these strains.

Experiments on Salmonella paratyphi-B subline SL 160 of strain SW 543 gave rather variable results. Sometimes (e.g. Expt. ix of Table 1) the numbers of motile descendants found in the progenies of 'initials' did not show a bimodal distribution. At other times (e.g. Expt. viii of Table 1) the results obtained were similar to those with SW 541. In pedigree experiments it was easy to demonstrate the unilinear transmission through many generations of ability to swim in broth, perhaps because in this strain motile organisms were less prone to get stuck to interfaces. Several attempts were made to obtain pedigrees of $E$ organisms in strain SL 160, but mostly without success; none of the re-isolated motile descendants of the 'initial' proving to be $E$. However, in one experiment 19 motile descendants of about the 12th generation were isolated, and one of them showed the $E$ character (c. 17 motile descendants).

\section{DISCUSSION}

\section{The evidence for unilinear transmission of two different characters}

The experiments described were undertaken to test the hypothesis of Stocker et al. (1953) that trail formation resulted from the unilinear transmission of motility amongst the progeny of organisms which had been incompletely or 'abortively' transformed. They have led to the conclusions: (i) that trails result from the unilinear transmission not of motility but of the ability to generate locomotor apparatus; (ii) that amongst the progeny of organisms which have generated such locomotor apparatus there is unilinear transmission of ability to travel in a liquid medium, though not in a semisolid one. These two unilinearly-transmitted characters have above been attributed to two hypothetical particles, the 'supernumerary gene' and the 'motilityconferring particle'; though the material basis of the two characters is uncertain this does not affect the validity of the conclusion that two characters are unilinearly transmitted. As this conclusion was unexpected and as the experiments of Lederberg (1956) do not unequivocally confirm it, the experimental data on which it is based have been given in some detail. The argument from the data to the hypothesis may be summarized.

Trails $2 \mathrm{~cm}$. or more long must mark the path through the agar of an organism, or rather of a succession of organisms. If the progeny of the organism which initiated the trail included at any time more than one descendant with the ability to form a trail, the trail would branch; the absence of branching therefore implies unilinear transmission of the character 'ability to produce a trail'.

The pedigree experiments show that, even after 20 generations, amongst the 
progeny of an organism made motile by abortive transduction there may be found one descendant, and one only, with the $E$ character (ability to produce $\geqslant 15$ motile descendants). This indicates unilinear transmission of the $E$ character. The pedigree experiments also show that the character 'motility in broth' may be unilinearly transmitted for many generations amongst the progeny of nori- $E$ motile organisms.

If it be accepted that the pedigrees establish the unilinear transmission of these two characters ( $E$ character and 'motility in broth') it must be decided whether one or both characters correspond to the unilinearly transmitted 'ability to form a trail'. Several lines of evidence indicate that organisms with unilinearly transmitted 'motility in broth' do not form trails. Bacteria known from their pedigrees to be of this kind did not produce trails when transferred to semisolid medium. Such organisms have been seen to divide and produce one daughter which was non-motile from the moment of fission; whereas it appears from the number of colonies initiated/generation-time in a trail that there is phenotypic delay in loss of motility when a trail-forming organism divides. Furthermore, most motile 'initials' isolated gave rise to several motile descendants which transmitted motility unilinearly, and if each such descendant could form a trail, several trails would start from a common origin; but in fact all or nearly all trails arise singly.

On the other hand, all the data are consistent with the hypothesis that the unilinearly-transmitted $\boldsymbol{E}$ character is the same as the unilinearly-transmitted ability to form a trail, and that both reflect unilinearly-transmitted ability to generate locomotor apparatus (MC particles). Parallel tests have shown that 'initials' with the two characters are present in about the same proportion in particular populations.

Lederberg (1956; and personal communication) in experiments with Salmonella paratyphi-B strain SW 543, subline SW 666, obtained results which, though not incompatible with the above hypothesis, yet do not unequivocally support it; and in a few experiments on strain SW 543, subline SL 160, I obtained generally similar results. In pedigree experiments on strain SW 543 it is easy to demonstrate unilinear transmission of motility in liquid medium but difficult or impossible to show that the $E$ character is transmitted unilinearly. It may be that in this strain the $E$ character is nearly always lost within the first few generations of unilinear transmission, e.g. by the loss or decay of the 'gene' presumed to account for it, or through the death of the organism containing it. However, the results obtained with other non-motile strains (Table 1, etc.) suggest that the conclusions as to abortive transduction drawn from experiments on Salmonella typhimurium SW 541 are generally applicable.

It has been argued above that only $E$ organisms can produce trails in the semisolid medium used. The experiments of Lederberg (1956) clearly show that several organisms amongst the progeny of an 'initial' can produce trails in a semisolid medium with a lower content of agar and gelatin than that of the 'standard' medium used in my experiments. This is not surprising, for in a medium with a sufficiently low gel content a bacterium which contained a single MC particle would presumably be motile, as it is in liquid medium. 


\section{The motility-conferring particle}

If the material basis of the unilinear transmission of 'motility in broth' is a motility-conferring particle, then such particles are presumably concerned in the motility of normal motile Salmonella strains also, and unilinear transmission of motility might be expected to occur in other situations where motile bacteria produce non-motile offspring. Mr C. Quadling (unpublished; see Stocker, 1956; Quadling \& Stocker, 1956a) demonstrated unilinear transmission of motility in two such situations: (i) in some non-flagellated Salmonella strains in which he found a very small proportion of motile organisms which were not mutants, since nearly all their progeny were non-motile; (ii) in some strains which were motile when grown at one temperature but on transfer to another temperature no longer produced flagella. Lederberg (1956) found unilinear transmission of motility in Salmonella dublin SW 553, another non-flagellated strain in which rare motile organisms occur.

It is generally believed that flagella are the locomotor organs of Salmonella, though this is not universally accepted (e.g. Pijper, 1951). This suggests that the MC particle may be a flagellum, or a particle (e.g. basal granule) which determines the production of a flagellum. This would account for the immediate non-motility of the daughter which does not receive the MC particle. On this interpretation salmonellas require several flagella to travel through the semisolid medium used, though one suffices for motility in broth.

A critical test of the relation between flagellum and MC partiele would be to determine, by staining or electron microscopy, the number of flagella on a bacterium inferred from its pedigree to be motile by possession of a single MC particle. Technical difficulties have prevented the performance of this experiment. However, Mr C. Quadling (personal communication) found that in Salmonella populations unable to make new flagella there was a good correlation between numbers of flagella seen in stained preparations, and numbers of MC particles inferred from pedigree experiments. This makes it seem probable that the MC particle is a flagellum, or a structure which determines the production of one.

If this be so, the pedigrees of non- $E$ organisms imply that the flagella of a dividing bacterium are being distributed about equally between its daughters. This is incompatible with the conclusion of Bisset (1951) who inferred, from electron micrographs and stained preparations, that in dividing Gram-negative bacilli the portion of the cell-wall bearing the parental flagella was retained in its entirety by one daughter, the other daughter growing new flagella. However recent observations (Quadling \& Stocker, 1956 b) on the distribution of numbers of flagella/bacterium in growing cultures in which no new flagella are being formed suggest that, at least in some circumstances, Bisset's conclusion is incorrect for Salmonella typhimurium.

It has been calculated above that the non- $E$ daughter of an $\boldsymbol{E}$ organism probably produces $c .15$ potentially motile progeny, and therefore probably received from its parent $c .15 \mathrm{MC}$ particles; this is considerably more than the average number of flagella/bacterium in a motile derivative of strain SW 541, 
as determined by staining the flagella by Leifson's method (1951) (Pl. 1, fig. 6). However, if the MC particle is a granule which secretes a flagellum and if there is some delay between the formation of the granule and its production of a visible flagellum then in a growing culture of a flagellated strain each organism would contain more $\mathrm{MC}$ particles than visible flagella, which would account for this discrepancy.

The experiments described above, together with those of Lederberg (1956) and of Mr C. Quadling (unpublished), indicate the occurrence of unilinear transmission of motility in all non-flagellated Salmonella strains tested, and also in Salmonella typhimurium SW 578, a flagellated but non-motile ('paralysed') strain. It seems that all these strains are non-motile because of a genetically-determined inability of their cells to make new MC particles, despite their ability to utilize and transmit to their progeny any MC particles which they receive from their parents. The genetic factors responsible for nonmotility in most of these strains are non-homologous (Stocker et al. 1953; Stocker \& Quadling, unpublished).

\section{The non-replicated supernumerary gene}

The only circumstance in which trails and, it is inferred, abortive transduction of motility, have been seen is when cultures of non-motile strains have been treated with phage lysates capable also of evoking swarms, i.e. of effecting complete transduction of motility. The first step in abortive transduction is, therefore, as in complete transduction, the bringing-in by a phage particle of something from the donor organism. Trails (and swarms) may be evoked from one non-motile strain by treatment with phage grown on another non-motile strain; therefore the particle transferred in abortive transduction cannot be anything which is present only in motile organisms, and is either a gene or a specific gene product. The unilinearly transmitted particle postulated to account for trail formation may then be either this same particle, or some product of its action, though not necessarily a specific product (Lederberg, 1956). However, the most economical hypothesis is that the particle transferred by phage is a gene, and that this gene itself is subsequently transmitted unilinearly. The best evidence that the particle is a gene would be to show that after unilinear transmission through many generations it sometimes began to multiply pari passu with the organisms, i.e. that an abortive transformation became complete. No evidence that this occurs has been met in pedigree experiments, and the evidence that swarms occasionally arise from trails is equivocal. The proof that the unilinearly transmitted particle which confers the $E$ and trail-forming characters is a gene, not a product of gene action, is thus incomplete. It might be said that a particle which is not replicated is by definition not a gene and it would perhaps be more precise to say that the particle concerned was a gene in the donor organism, before its transfer to the recipient.

Mr H. Ozeki (personal communication) has recently obtained evidence of abortive transduction of prototrophy to certain purine-dependent mutants of 
Salmonella typhimurium; his conclusions as to the mechanism are similar to those presented here.

Though the postulated particle has been called a gene, it probably consists of a fragment of a genetic organelle, perhaps a piece of a linear chromosomelike structure. Some evidence for this is given by observations (Lederberg, 1956; Stocker, unpublished) on Salmonella paratyphi-B strain SW 543. The gene determining absence of flagella in this strain is linked to a gene which regulates the antigenic character of the flagella (Stocker et al. 1953). The immobilizing action of antiflagellar sera on abortively transformed bacteria suggests that in this strain the non-replicated genetic particle always or nearly always contains both the gene for formation of flagella and the linked gene for flagellar antigenic type; whereas the fragment incorporated into the gene-set in complete transformation frequently contains only the former gene.

The ratio of trails to swarms varies widely from one pair of donor and recipient strains to another; it is not known what determines it. Partial non-homology of the genetic material with that of the recipient might hinder its incorporation into the genetic organelle; but this cannot be the only cause of non-incorporation, for a non-motile strain treated with a lysate of a motile variant derived from it by mutation produces trails as well as swarms. Perhaps some phage-borne genetic particles are of such a kind that they cannot be incorporated into the genetic organelle; or perhaps incorporation or its failure is only decided after the particle enters the recipient. If the latter is the case, it seems that the genetic material, if not at once incorporated, soon becomes incapable of incorporation. This might result either from some decay of the particle or from its becoming lodged in some special situation. Stocker (1953) suggested that transformation of pneumococci and transduction in Salmonella both resulted from the introduction into the organism of genetically active deoxyribonucleic acid (DNA), its side-by-side pairing with homologous material in a linear genetic organelle, and its consequent incorporation in continuity in a replica organelle in process of formation alongside the original one. On this model a failure of incorporation of part of an introduced fragment might result if one end of it became apposed to its homologue, and in consequence incorporated into a replica 'chromosome', while the other end, through failure to become apposed in time, remained as a side-arm when formation of the replica organelle was completed. If replication (or separation of replica from original) affected only genetic material arranged along the main length of the genetic organelle, then the side-arm would not be replicated, and would consequently be transmitted unilinearly. If a gene for formation of MC particles was carried on such a side-arm, abortive transduction of motility would be accounted for.

\section{Unilinear transmission of characters}

Investigation of abortive transduction of motility in Salmonella has revealed two instances of unilinear transmission of characters. When a unicellular (acellular) organism (or cell of a multicellular one) divides, the substance of the parent cell is distributed, equally or unequally, amongst the daughters; it 
would therefore appear that all stable particulate cell structures and substances must be transmitted down one line of descent. There are, however, few reports of unilinear transmission of characters. This is presumably because the unilinear transmission of a particle will not produce striking effects if (i) the descendants not receiving the original particle synthesize new particles indistinguishable from it, or (ii) if the presence of a single particle (e.g. a protein molecule) in a cell is unrecognizable. In Paramecium the parental contractile vacuoles are transmitted to the daughters; but since each daughter also synthesizes new vacuoles indistinguishable from the old ones, the unilinear transmission of the parental vacuole is not observable. In bacteria there is evidence that molecules of an adaptive (inducible) enzyme are transmitted to the progeny even when synthesis has ceased owing to withdrawal of the inducer; each molecule of enzyme is presumably transmitted unilinearly, but this cannot be tested since the presence of a single enzyme molecule in a bacterium cannot be recognized.

Several instances of unilinear transmission have, however, been described. In a pedigree recorded by Jennings (1908) division of a misshapen Paramecium gave rise to a daughter with an abnormal protrusion or 'spine', whose unilinear transmission was observed for twenty generations. Jacob (1954) and Bertani (1954) found that the genes of a phage particle absorbed by a bacterium already lysogenic for a related phage persisted, but did not multiply, during several generations of bacterial growth. Lederberg (1956) described the unilinear transmission of formazan granules in Salmonella.

In many unicellular (or acellular) organisms (e.g. the yeasts) division is unequal, in that one daughter retains the parental cell wall; as might be expected, unilinear transmission of morphological characters has been observed in such organisms (Jennings, 1929; other instances are cited by Lederberg, 1956)

I wish to thank Dr C. Quadling and Mr H. A. Milne for preparing and photographing material used for illustrations.

\section{REFERENCES}

Barer, R. \& Saunders-Singer, A. E. (1948). A new single-control micro-manipulator. Quart. F. micr. Sci. 89, 439.

Bertani, G. (1954). Studies on lysogenesis. III. Superinfection of lysogenic Shigella dysenteriae with temperate mutants of the carried phage. J. Bact. 67, 696.

Bisset, K. A. (1951). The development of the surface structures in dividing bacteria. J. gen. Microbiol. $5,155$.

de Fonbrune, P. (1949). Technique de Micromanipulation. Paris: Masson.

$\mathrm{J}_{\mathrm{ACOB}}, \mathrm{F}$. (1954). Les bactéries lysogènes et la notion de provirus. Paris: Masson.

Jennings, H. S. (1908). Heredity, variation and evolution in Protozoa. I. The fate of new structural characters in Paramecium, in connection with the problem of the inheritance of acquired characters in unicellular organisms. J. exp. Zool. 5, 577.

Jennings, H. S. (1929). Genetics of the Protozoa. Bibliogr. genet. 5, 103.

Lederberg, J. (1956). Linear inheritance in transductional clones. Genetics (in the Press). 
Lederberg, J. \& Edwards, P. R. (1953). Serotypic recombination in Salmonella. J. Immunol. 71, 232.

LEIfson, E. (1951). Staining, shape, and arrangement of bacterial flagella. J. Bact. $62,377$.

PiJPer, A. (1951). Bacterial flagella. Nature, Lond. 168, 749.

Quadling, C. \& Stocker, B. A. D. $(1956 a)$. The occurrence of motile cells, which are not mutants, in some Salmonella $O$ strains. J. gen. Microbiol. 14, i.

Quadling, C. \& Stocker, B. A. D. (1956b). An environmentally induced transition from the flagellated to the non-flagellated state in Salmonella; the fate of parental flagella at cell division. J. gen. Microbiol. 15, i.

Stocker, B. A. D. (1953). Genetic transduction in bacteria. Atti VI Congr. int. Microbiol. 1, 671.

Stocker, B. A. D. (1956). Bacterial flagella: morphology, constitution and inheritance. In Bacterial Anatomy. Symp. Soc. gen. Microbiol. 6, 19.

Stocker, B. A. D., Zinder, N. D. \& Lederberg, J. (1953). Transduction of flagellar characters in Salmonella. J. gen. Microbiol. 9, 410.

Zinder, N. D. \& Lederberg, J. (1952). Genetic exchange in Salmonella. J. Bact. 64,679 .

\section{EXPLANATION OF PLATE}

Fig. 1. Trails produced by Salmonella paratyphi-B strain SW 966, treated with lysate of $S$. typhimurium strain TM 2; the treated organisms were plated at the centre of a $5 \mathrm{~cm}$. Petri dish of semisolid medium, which was incubated at $37^{\circ}$ for $42 \mathrm{hr} . \times 1$.

Fig. 2. Distal (growing) end of one of the trails shown in Fig. $1 . \times 8$.

Fig. 3. Droplet containing hundreds of non-motile and one motile organism, which has produced a streak (indicated by arrow) during 10 sec. exposure; dark-ground illumination. $\times c .300$.

Fig. 4. As fig. 3, but droplet contains one motile and thousands of non-motile organisms (smaller than those in fig. 3 because not in log phase). $\times c .300$. (In figs. 3 and 4 the non-motile bacteria appear blurred, owing to Brownian movement.)

Fig. 5. Trail produced by a motile 'initial' isolated from a lysate-treated culture of strain SW 541 and incorporated in a pour-plate of semisolid medium, which was incubated at $37^{\circ}$ for $18 \mathrm{hr}$. $\times c .10$.

Fig. 6. Organisms of a motile variant obtained from Salmonella typhimurium strain SW 541 by transduction; flagella stained by the method of Leifson (1951). $\times$ c. 650 . 
Journal of General Microbiology, Vol. 15, No.3
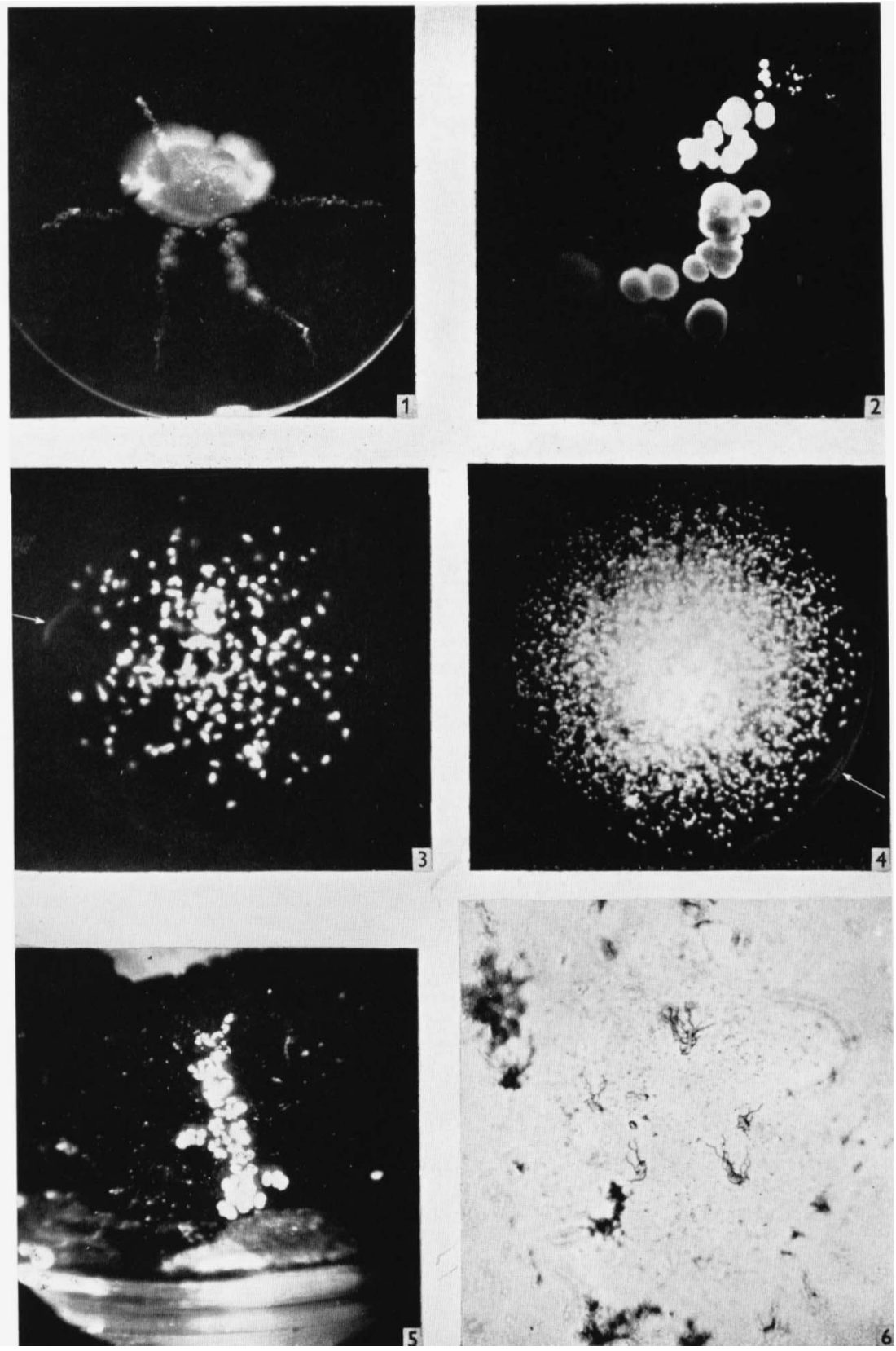

B. A. I). Stocker-Abortive trinsteften of mothlity. Plate 1 